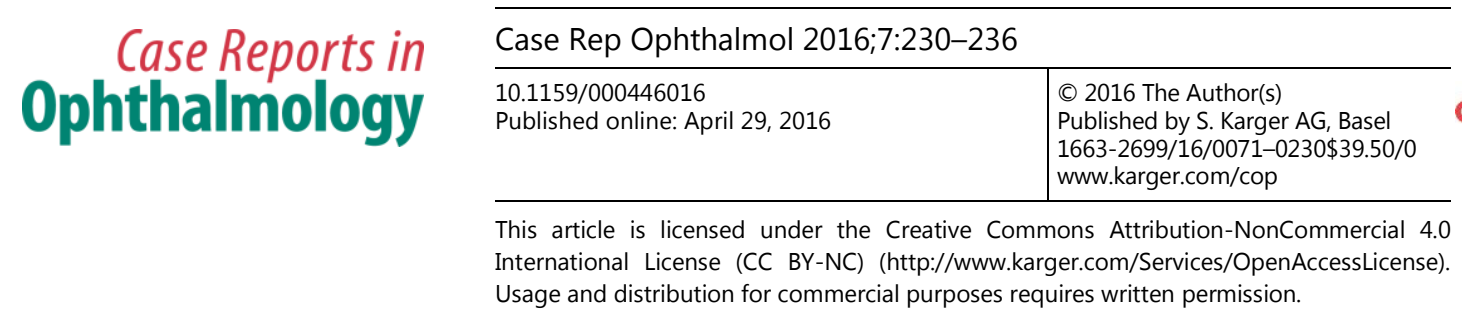

\title{
A Case of Sustained Intraocular Pressure Elevation after Multiple Intravitreal Injection of Ranibizumab and Aflibercept for Neovascular Age-Related Macular Degeneration
}

\author{
Hisashi Matsubara Ryohei Miyata Maki Kobayashi \\ Hideyuki Tsukitome Kengo Ikesugi Mineo Kondo \\ Department of Ophthalmology, Mie University Graduate School of Medicine, Tsu, Japan
}

\section{Keywords}

Age-related macular degeneration - Anti-vascular endothelial growth factor · Ranibizumab * Aflibercept · Multiple intravitreal injections · Medication switching · Intraocular pressure . Ocular hypertension

\begin{abstract}
Intravitreal injections of anti-vascular endothelial growth factor (VEGF) agents are widely used to treat neovascular age-related macular degeneration (nAMD). Although these treatments are effective, multiple injections have recently been recommended to ensure that there is a good long-term prognosis. However, sustained intraocular pressure (IOP) elevations have been reported to develop after multiple injections of anti-VEGF agents. We present our findings of a case of uncontrolled and persistent IOP elevation after switching from intravitreal ranibizumab injections to intravitreal aflibercept injections. A 74-year-old Japanese man without a history of glaucoma underwent 22 ranibizumab injections for nAMD and suddenly developed an elevated IOP after the 22nd injection. Although the subsequent medical treatment led to normalization of his IOP, the subretinal fluid under the central fovea remained even after the 25th injection of ranibizumab. Thus, ranibizumab treatment was switched to bimonthly intravitreal aflibercept injections in conjunction with glaucoma medi-
\end{abstract}


cations. His IOP recovered to within the normal range; however, after the 11th aflibercept injection, there was a sudden elevation of his IOP in spite of the continued glaucoma medications. Due to this sustained IOP elevation, his aflibercept injections were suspended for 16 weeks. Because his IOP could not be normalized by a full glaucoma medication regimen, the patient underwent trabeculotomy, which resulted in a lowering of the IOP to normal levels. We conclude that patients who receive serial intravitreal injections of anti-VEGF agents need to be closely monitored because severe and sustained ocular hypertension can develop.

(C) 2016 The Author(s)

Published by S. Karger AG, Basel

\section{Introduction}

Intravitreal injections of anti-vascular endothelial growth factor (VEGF) agents are commonly used to treat various intraocular vascular diseases. Because of the effectiveness of these treatments, they have become the first-line therapy for neovascular age-related macular degeneration (nAMD). Although intravitreal injections of anti-VEGF agents are effective, some studies have reported intraocular pressure (IOP) elevations after anti-VEGF injections. There is immediate IOP elevation following intravitreal injections; however, the elevations are transient and related to the increase in the intraocular volume [1].

On the other hand, persistent IOP elevations have been reported as a rare complication in case reports and in a case series following intravitreal ranibizumab or bevacizumab injections $[2,3]$. Because the IOPs in some cases could not be controlled by topical glaucoma drugs, glaucoma operations were required in these patients [4]. In the results of the 2015 VEGF Trap-Eye: Investigation of Efficacy and Safety in Wet AMD (VIEW) trial, the proportion of eyes with an IOP $>21 \mathrm{~mm} \mathrm{Hg}$ after ranibizumab serial injections at 4-week intervals was $20.2 \%$, and it was $12.1 \%$ after aflibercept injections at 8 -week intervals [5]. This report proposed that there was a lower possibility of sustained IOP elevations when aflibercept is used. Another study reported a decrease in the IOP in eyes that were switched to aflibercept after undergoing ranibizumab injections [6]. However, there has been no report on uncontrolled persistent IOP elevations after patients were switched from intravitreal injections of ranibizumab to aflibercept.

Thus, the purpose of this report is to present our findings in a patient who developed a persistently high IOP elevation after being switched from repeated ranibizumab to aflibercept injections.

\section{Case Report}

A 74-year-old man with nAMD in his left eye was referred to our clinic. He had no history of glaucoma, ocular hypertension, or IOP asymmetry. Ophthalmic examinations showed a decimal best-corrected visual acuity (BCVA) of 0.4 in his left eye and of 1.2 in his right eye. Mild nuclear sclerosis cataracts and IOPs of $16 \mathrm{~mm} \mathrm{Hg}$ with open anterior chamber angle were found in both eyes. Fundus examination showed that there were no glaucomatous changes, but that there was subretinal hemorrhage near the fovea. Both fluorescein and indocyanine green angiography demonstrated the presence of the occult type of choroidal neovascularization (CNV) in the left eye. However, the patient stated that he did not wish to receive any therapy in his left eye for the nAMD. 


\section{Case Reports in Ophthalmology}

Two years later, optical coherence tomography (OCT) detected a CNV in his right eye (fig. 1). The BCVA was 1.0, and the IOP was $18 \mathrm{~mm} \mathrm{Hg}$. Therefore, we decided to start ranibizumab injections in his right eye. After 3-monthly ranibizumab injections for the induction phase, he received ranibizumab injections at 4- and 8-week intervals using a pro re nata protocol. During 27 months of treatment, the patient had received 22 ranibizumab injections in his right eye, and the IOP remained $<20 \mathrm{~mm} \mathrm{Hg}$ at all his visits. No ranibizumab treatment was performed in his left eye, and the IOP remained at approximately $15 \mathrm{~mm} \mathrm{Hg}$. Four weeks after the 22nd ranibizumab injection, the IOP of his right eye increased to $28 \mathrm{~mm} \mathrm{Hg}$, and subretinal fluid (SRF) was detected in the OCT images. He received his 23rd ranibizumab injection on the same day, and he began receiving $0.005 \%$ latanoprost and $0.5 \%$ timolol once a day to treat the ocular hypertension. Follow-up examinations were performed at 2- and 4week intervals after the 23rd ranibizumab injection, and they demonstrated that the IOP had decreased to 17-19 $\mathrm{mm} \mathrm{Hg}$. However, the OCT examination performed at 4 weeks after the 23rd ranibizumab injection showed that the SRF was still present under the central fovea. As a result, the patient received two additional ranibizumab injections at 4-week intervals for the 24th and 25th treatments. Because the size of the SRF had not decreased at 4 weeks after the 25th ranibizumab injection, ranibizumab was stopped and the patient was switched to intravitreal aflibercept injections (fig. 2). At all subsequent aflibercept treatments, the patient underwent paracentesis prior to the aflibercept injections. Before the first aflibercept injection, the IOP of the right eye was $24 \mathrm{~mm} \mathrm{Hg}$, and 2 weeks later, both the SRF and the retinal edema had completely disappeared. However, the IOP was still high at $26 \mathrm{~mm} \mathrm{Hg}$. Therefore, $0.005 \%$ latanoprost was changed to $0.03 \%$ bimatoprost, and $1 \%$ dorzolamide was added 3 times/day. Two weeks later, the IOP had decreased to $15 \mathrm{~mm} \mathrm{Hg}$. To ensure that there would be no retinal exudation, we performed aflibercept injections bimonthly. The follow-up examinations revealed the presence of a dry retina, and the use of $0.03 \%$ bimatoprost and 1\% dorzolamide maintained the IOP under $20 \mathrm{~mm} \mathrm{Hg}$ until the 11th aflibercept injection. Four weeks after the 11th aflibercept injection, there was an IOP elevation to $30 \mathrm{~mm} \mathrm{Hg}$ without any other signs of changes in the vitreous cavity and anterior chamber. Therefore, $0.1 \%$ brimonidine was added twice a day. Two weeks later, the IOP was $22 \mathrm{~mm}$ $\mathrm{Hg}$, and at 2 weeks after the last examination, the retina remained dry but the IOP was 27 $\mathrm{mm} \mathrm{Hg}$. Therefore, it was decided to stop all further aflibercept injections and to begin administering a $250-\mathrm{mg} /$ day dose of acetazolamide orally. Because the IOP was still $25 \mathrm{~mm} \mathrm{Hg}$ 3 weeks later, the oral acetazolamide dose was increased to $500 \mathrm{mg} /$ day. Unfortunately, 4 weeks later, which was 16 weeks after the last (11th) aflibercept treatment, the IOP was still $26 \mathrm{~mm} \mathrm{Hg}$, even though antiglaucoma medications and acetazolamide had been continued to be administered.

It was decided that a trabeculotomy was needed for the right eye. Two weeks after surgery, the IOP had decreased to $15 \mathrm{~mm} \mathrm{Hg}$ without the use of any antiglaucoma medications (fig. 3). We used 1.5\% topical levofloxacin for 3 days before and after each injection, and we never used a topical steroid after the injections. During the entire follow-up period of 56 months, no inflammatory cells were observed in the anterior chamber of either eye, and the IOP of the left eye remained approximately $15 \mathrm{~mm} \mathrm{Hg}$. The BCVA in the right eye was 0.5 because of a mild macular atrophy. 


\section{Case Reports in Ophthalmology}

Case Rep Ophthalmol 2016;7:230-236

Matsubara et al: A Case of Sustained IOP Elevation after Multiple Intravitreal Injection of Ranibizumab and Aflibercept for Neovascular Age-Related Macular Degeneration

\section{Discussion}

Earlier studies have reported that IOP elevations are observed not only in patients with pre-existing glaucoma but also in patients without pre-existing glaucoma [2, 3]. This suggests that sustained IOP elevations might be associated with the multiple anti-VEGF agent injections. Even so, the exact mechanism responsible for the sustained IOP elevation has yet to be determined.

In our case, the right eye had an open anterior chamber angle, there was no history of glaucoma susceptibility or ocular signs of glaucoma, and there was no IOP asymmetry prior to the start of the ranibizumab treatments. In addition, ranibizumab injections have been performed approximately every 4 weeks while the aflibercept injections have been performed bimonthly, and there was no elevation of the IOP in the right eye during the initial 21 repeated ranibizumab injections and the 10 bimonthly aflibercept injections while using glaucoma medications. Subsequently, without any indication, there was a sudden IOP elevation immediately after the $22 \mathrm{nd}$ ranibizumab and the 11 th aflibercept injection. This pattern of a sudden elevation of the IOP following the multiple anti-VEGF agent injections is very similar to that of past reports. Due to the lack of any pre-existing signs of glaucoma, these findings strongly suggest that there is an association between the persistent IOP elevations and the multiple serial injections of the anti-VEGF drugs. Past studies have proposed that there was a lower possibility of sustained IOP elevations when aflibercept is used [5, 6]. Although we were able to achieve an IOP $<20 \mathrm{~mm} \mathrm{Hg}$ by changing the type of glaucoma medication after the patient was switched from ranibizumab to aflibercept, the IOP increased to $>25 \mathrm{~mm} \mathrm{Hg}$ after we began the serial aflibercept injections. Moreover, we were not able to achieve any reduction in the IOP elevation by switching from ranibizumab to aflibercept or by discontinuing long-term aflibercept injections for a period of 16 weeks. Therefore, we suggest that the sustained IOP elevation would have occurred regardless of the type of antiVEGF drugs, and that it would not have been restored by either switching or stopping the anti-VEGF injections. The reasons for the sustained elevation in our case might have been due to irreversible changes that occurred at the time when the patient was switched from ranibizumab to aflibercept, or it might be that aflibercept could have remained in his right eye even after we had discontinued the aflibercept injection for 16 weeks, thereby affecting the IOP. In fact, it is well known that aflibercept can completely suppress VEGF activity in the aqueous humor for a mean duration of 71 days [7], and for 80 days in the vitreous [8]. However, because individual differences may exist with regard to how long aflibercept can suppress VEGF [8], we cannot conclude whether aflibercept had been washed out in our patient's right eye during the 16 weeks after we discontinued the aflibercept injections. On the other hand, significant inflammatory reactions following intravitreal injection of bevacizumab and sustained IOP elevation and keratic precipitates in the trabecular meshwork have been reported after multiple injections of both bevacizumab and ranibizumab $[9,10]$. Based on these findings, it has been proposed that IOP elevation may be triggered by a physical blockage of the outflow by the inflammatory reaction and the subsequent trabeculitis. Although we did not find any inflammatory cells or any other reaction that occurred after either the ranibizumab or aflibercept injections, we believe that these inflammatory reactions were possible mechanisms for the sustained IOP elevation in our case. We cannot completely rule out the possibility of either a subclinical immune response or the presence of immunocomplexes that occluded the trabecular meshwork and obstructed the aqueous flow. In our case, the multiple and periodic injections of ranibizumab and aflibercept ensured that there was a regular supply available prior to being washed out from the anterior chamber, 
week discontinuation period caused by residual aflibercept.

Bimonthly aflibercept injections are commonly performed and widely used. Thus, it is quite possible that there will be an increase in the number of sustained IOP elevations that are observed after anti-VEGF agent injections. Therefore, patients need to be closely followed for sustained IOP elevation because if untreated, it can lead to optic nerve damage. Further studies investigating the incidence of this potential side effect will need to be undertaken to confirm these findings. In addition, it will be necessary to keep medical practitioners and clinicians informed on the need for close long-term surveillance of IOP after antiVEGF agent injections to ensure safe and beneficial treatments.

\section{Conclusions}

Our findings show that persistent IOP elevation can occur after switching from repeated intravitreal ranibizumab to intravitreal aflibercept injections. Thus, clinicians should monitor patients who are being treated with multiple anti-VEGF agents more frequently.

\section{Acknowledgements}

The authors thank Prof. Duco Hamasaki of the Bascom Palmer Eye Institute, University of Miami, for the critical discussion and final manuscript revisions.

\section{Statement of Ethics}

The patient provided written informed consent for submission of the case report and any accompanying images. This study was approved by the Ethics Committee of Mie University Graduate School of Medicine, Tsu City, Mie, Japan.

\section{Disclosure Statement}

The authors declare that they have no conflicts of interest.

\section{References}

1 Bakri SJ, Pulido JS, McCannel CA, Hodge DO, Diehl N, Hillemeier J: Immediate intraocular pressure changes following intravitreal injections of triamcinolone, pegaptanib, and bevacizumab. Eye (Lond) 2009;23:181-185.

-2 Bakri SJ, McCannel CA, Edwards AO, Moshfeghi DM: Persistent ocular hypertension following intravitreal ranibizumab. Graefes Arch Clin Exp Ophthalmol 2008;246:955-958.

- 3 Adelman RA, Zheng Q, Mayer HR: Persistent ocular hypertension following intravitreal bevacizumab and ranibizumab injections. J Ocul Pharmacol Ther 2010;26:105-110.

-4 Yu AL, Seidensticker F, Schaumberger M, Welge-Lussen U, Wolf A: Evaluation of intraocular pressure elevation after multiple injections of intravitreal ranibizumab. Clin Ophthalmol 2014;8:743-747.

-5 Freund KB, Hoang QV, Saroj N, Thompson D: Intraocular pressure in patients with neovascular agerelated macular degeneration receiving intravitreal aflibercept or ranibizumab. Ophthalmology 2015;122:1802-1810. 


\section{Case Reports in \\ Ophthalmology}

\begin{tabular}{l|l}
\hline $10.1159 / 000446016$ & $\begin{array}{l}\text { (c) } 2016 \text { The Author(s). Published by S. Karger AG, Basel } \\
\text { www.karger.com/cop }\end{array}$ \\
\hline
\end{tabular}

Matsubara et al: A Case of Sustained IOP Elevation after Multiple Intravitreal Injection of Ranibizumab and Aflibercept for Neovascular Age-Related Macular Degeneration

-6 Rusu IM, Deobhakta A, Yoon D, Lee M, Slakter JS, Klancnik JM, Thompson D, Freund KB: Intraocular pressure in patients with neovascular age-related macular degeneration switched to aflibercept injection after previous anti-vascular endothelial growth factor treatments. Retina 2014;34:21612166.

7 Fauser S, Schwabecker V, Muether PS: Suppression of intraocular vascular endothelial growth factor during aflibercept treatment of age-related macular degeneration. Am J Ophthalmol 2014;158:532536.

-8 Stewart MW, Rosenfeld PJ, Penha FM, Wang F, Yehoshua Z, Bueno-Lopez E, Lopez PF: Pharmacokinetic rationale for dosing every 2 weeks versus 4 weeks with intravitreal ranibizumab, bevacizumab, and aflibercept (vascular endothelial growth factor Trap-eye). Retina 2012;32:434-457.

-9 Georgopoulos M, Polak K, Prager F, Prünte C, Schmidt-Erfurth U: Characteristics of severe intraocular inflammation following intravitreal injection of bevacizumab (Avastin). Br J Ophthalmol 2009;93:457462.

10 Sniegowski M, Mandava N, Kahook MY: Sustained intraocular pressure elevation after intravitreal injection of bevacizumab and ranibizumab associated with trabeculitis. Open Ophthalmol J 2010;22:28-29.
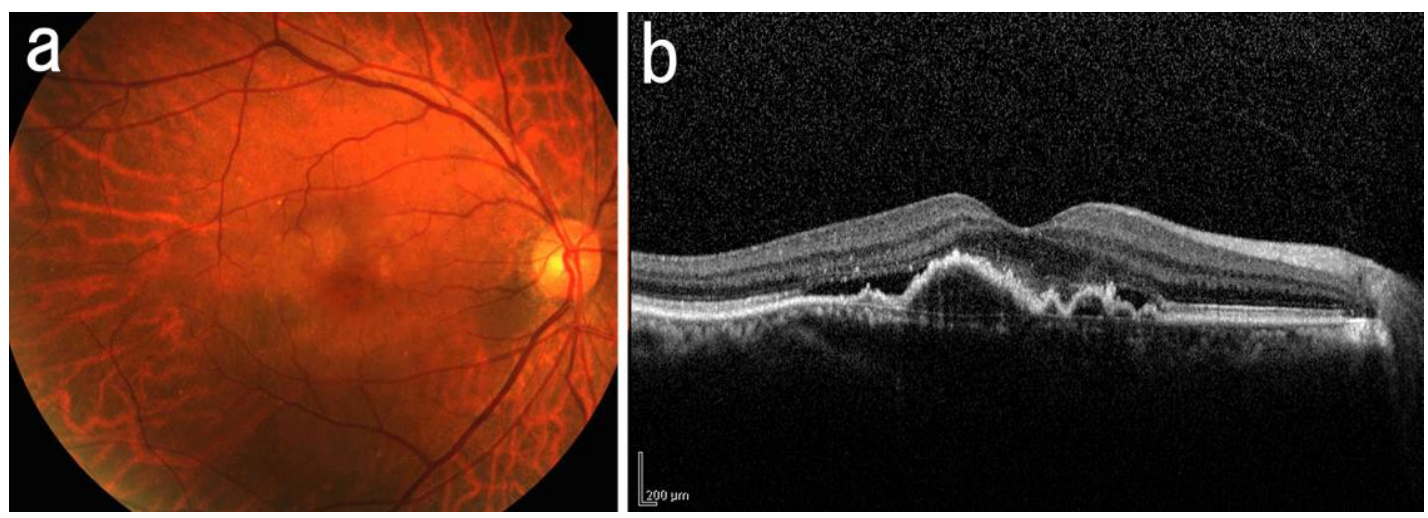

Fig. 1. Fundus photograph and OCT image of a patient with nAMD in the right eye. a Fundus photograph before the first intravitreal ranibizumab injection showing normal optic disc cupping. b OCT image showing the presence of CNV and SRF. 


\section{Case Reports in Ophthalmology}

\begin{tabular}{l|l}
\hline Case Rep Ophthalmol 2016;7:230-236 \\
\hline $10.1159 / 000446016$ & $\begin{array}{l}\text { c } 2016 \text { The Author(s). Published by S. Karger AG, Basel } \\
\text { www.karger.com/cop }\end{array}$ \\
\hline
\end{tabular}

Matsubara et al.: A Case of Sustained IOP Elevation after Multiple Intravitreal Injection of Ranibizumab and Aflibercept for Neovascular Age-Related Macular Degeneration
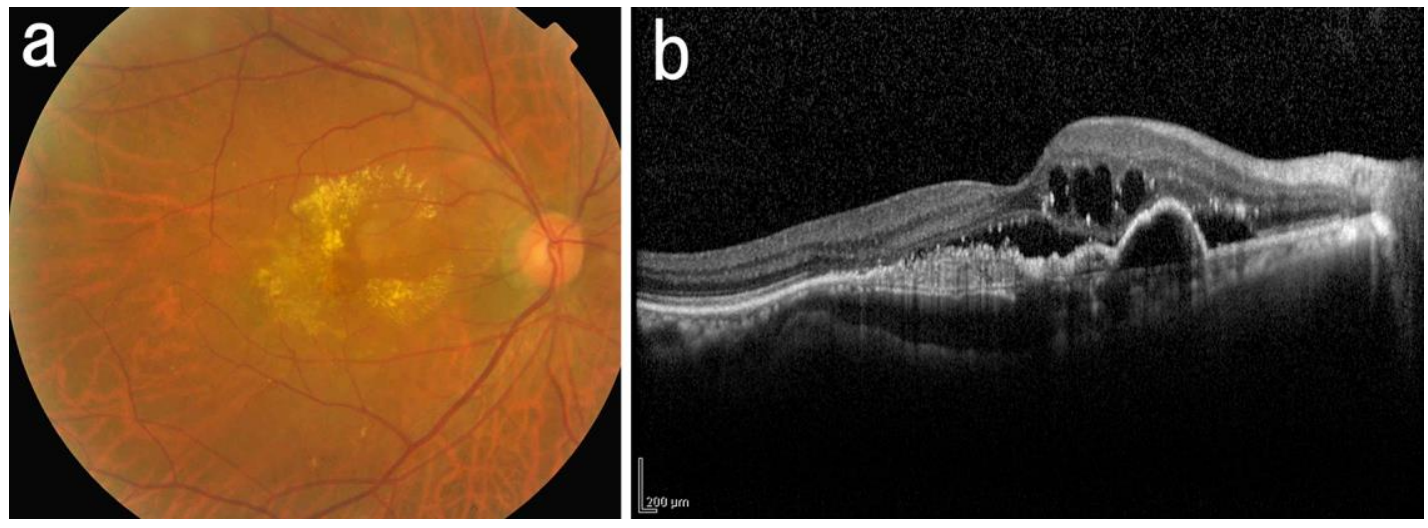

Fig. 2. Fundus photograph and OCT image of the same patient with nAMD. a Fundus photograph at 4 weeks after the 25th intravitreal ranibizumab injection, and before the first intravitreal aflibercept injection showing the presence of hard exudates. b OCT image showing the residual SRF and retinal edema.
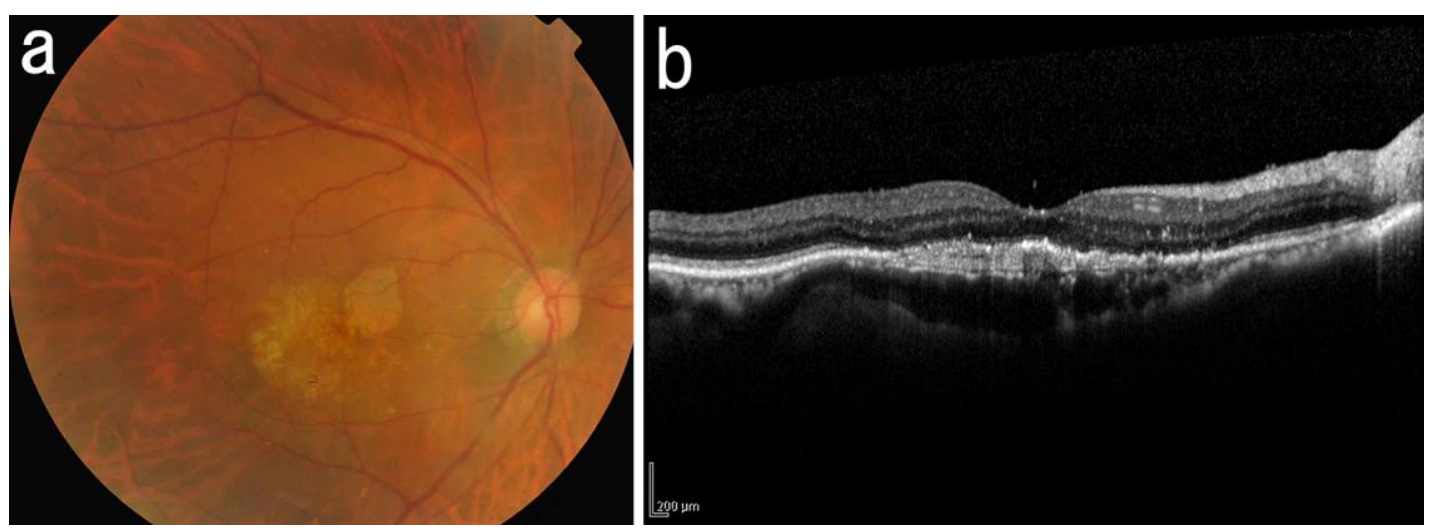

Fig. 3. Fundus photograph and OCT image of the same patient with nAMD. a Fundus photograph after trabeculotomy, demonstrating the absence of hard exudates. $\mathbf{b}$ OCT image after trabeculotomy showing that both the SRF and the retinal edema have completely disappeared. 